\title{
A systematic analysis reveals gene expression alteration of serum deprivation response (SDPR) gene is significantly associated with the survival of patients with cancer
}

\author{
YINGSHUANG WANG ${ }^{1}$, ZHEN SONG $^{1}$, PING LENG $^{1}$ and YUN LIU ${ }^{2}$ \\ ${ }^{1}$ College of Medical Technology, Chengdu University of Traditional Chinese Medicine, Chengdu, Sichuan 611137; \\ ${ }^{2}$ Peking University Medical and Health Analytical Center, Peking University Health Science Center, \\ Beijing 100191, P.R. China
}

Received January 18, 2019; Accepted June 18, 2019

DOI: $10.3892 /$ or.2019.7212

\begin{abstract}
Serum deprivation response (SDPR) gene has been recently characterized as a gene signature marker or serving a tumor suppressor role in specific types of cancer. However, gene expression alterations of $S D P R$ in various types of cancer and their relevance to clinical outcomes remain unclear. In the present study, SDPR expression was profiled using the Oncomine database, and SDPR downregulation was indicated in most types of cancer. In agreement with previously reported breast cancer cases, downregulation of $S D P R$ was indicated to be significantly associated with poor survival in patients with lung cancer, glioma and sarcoma. To clarify why SDPR expression was altered in these types of cancer, both DNA methylation patterns and potential transcriptional factors of $S D P R$ were analyzed. Altered DNA methylation levels of SDPR were found in 17/18 cancer types using MethHC. To the best of our knowledge, the present study for the first time, identified the CpG site (cg10082589) as one of the best survival methylation markers for lung adenocarcinoma, and the $\mathrm{CpG}$ site (cg07488576) for sarcoma using Methsurv. In addition, GATA binding protein 2 was identified as a potential transcription factor for $S D P R$, by integrating and analyzing both the co-expressed genes and the potential transcription factor binding sites of SDPR. In the present study, the systematic analysis of $S D P R$ provided insight for the underlying
\end{abstract}

Correspondence to: Dr Yingshuang Wang, College of Medical Technology, Chengdu University of Traditional Chinese Medicine, 1166 Liutai Road, Chengdu, Sichuan 611137, P.R. China

E-mail: wangyingshuang@cdutcm.edu.cn

Dr Yun Liu, Peking University Medical and Health Analytical Center, Peking University Health Science Center, 38 Xueyuan Road, Beijing 100191, P.R. China

E-mail: 6250@bjmu.edu.cn

Key words: serum deprivation response, caveolae associated protein 2, gene expression alteration, survival analysis, gene methylation molecular mechanisms in cancer progression, revealing novel perspectives for the clinical prognostic evaluation of lung adenocarcinoma and sarcoma.

\section{Introduction}

Cancer has contributed to the rising rate in disease-associated mortality in the last few decades. The demand for early-stage diagnosis, prognosis and therapeutic targets is increasing. The serum deprivation response $(S D P R)$ gene has been characterized to serve a critical role in breast cancer as a tumor suppressor $(1,2)$, and recently showing a characteristic gene signature in specific types of cancer, including oral cancer, thyroid cancer and liposarcoma (3-5).

$S D P R$ localizes to chr2q 32-33, also known as caveolae associated protein 2 , and has been known for its role in caveolae formation (6-8). Its encoding protein SDPR, which is overexpressed in serum starved cells, was firstly identified as a substrate for protein kinase $\mathrm{C}$ (PKC) phosphorylation, an interaction that targets PKC in caveolae formation (9). Caveolae are plasma membrane microdomains involved in multiple biological processes, including lipid metabolism, endocytosis, cellular signal transduction, cell proliferation and migration $(7,10)$.

Previous studies have gradually implicated the differential expression and tumor suppressor function of $S D P R$ in cancer progression and metastasis; reduced $S D P R$ expression has been observed in breast $(1,2,11-13)$, kidney $(11,14)$ and prostate $(11,15)$ cancer. In breast cancer, SDPR was identified as a tumor suppressor $(1,2)$. SDPR serves an anti-metastatic function by promoting apoptosis (1), and depletion of SDPR induced epithelial-mesenchymal transition (EMT) through transforming growth factor- $\beta$ (TGF- $\beta$ ) signaling activation (2). The reduction of $S D P R$ expression has been reported to be associated with significantly reduced survival in patients with breast cancer, who underwent therapy (1).

In addition to breast cancer, it has been suggested that $S D P R$ may serve as a tumor suppressor gene, with a broader clinical relevance, in other types of cancer. In oral cancer, it was identified that $S D P R$-negative patients had high tumor progression (5), whereas in sarcoma (SARC), a lower $S D P R$ 
expression was observed in more aggressive or dedifferentiated tumor forms (4). In addition, it was suggested that the differently expressed SDPR gene can be used as a possible diagnostic marker to discriminate malignant tumors from benign formations, not only in serum from patients with kidney tumors (14), but also in follicular thyroid carcinomas (3). Nevertheless, gene expression alterations of SDPR in various types of cancer and their relevance to clinical outcomes remain unclear.

In the present study, mRNA levels of $S D P R$ were compared in various unique tumor tissue datasets compared with normal tissue datasets, indicating that $S D P R$ was downregulated in various types of cancer. In addition, downregulated $S D P R$ was found to be significantly associated with the survival of the patients, not only in previously reported breast cancer cases, but also in brain, lung and soft tissue tumors. However, SDPR failed to emerge as a frequent target for gene mutational inactivation in a previous next-generation sequencing study (16). As $S D P R$ has been reported to be hypermethylated and silenced in breast cancer cell lines, it was suggested that it is likely to be epigenetically inactivated in cancer (1). In order to examine the mechanism underlying SDPR downregulation in cancer, the present study analyzed and found SDPR gene methylation alteration between cancer and normal tissues. Furthermore, the present study investigated the methylation sites relevant to the survival of patients with lung adenocarcinoma (LUAD) and SARC. In addition, the potential transcription factor binding sites of the SDPR promoter were analyzed. The potential transcription factor GATA binding protein 2 (GATA2) was identified from the analysis of genes that are co-expressed with SDPR. The results of the present study provide additional insight in understanding the underlying molecular mechanisms of $S D P R$ in cancer, in addition to revealing a novel approach in the clinical prognostic evaluation and treatment of LUAD and SARC.

\section{Materials and methods}

Gene expression analysis using oncomine platform. The profile of $S D P R$ gene expression level in various types of cancer was identified in Oncomine ${ }^{\mathrm{TM}}$ Platform and the detailed datasets are available online (https://www.oncomine.org/) (17). By comparing with normal tissues, the mRNA expression-fold of SDPR in cancer tissues was obtained using the parameters of $\mathrm{P}$-value $<10^{-4}$ or 0.01 , fold-change $>2$, and gene ranking in the top $10 \%$. To adjust the false discovery rate, the $\mathrm{P}$-values were corrected by using the Benjamini-Hochberg procedure (B-H method) (18) in $\mathrm{R}$, version 3.5.0 (https://www.r-project.org/).

Prognoscan database analysis. The association between SDPR expression and survival in different types of cancer was analyzed using the PrognoScan database (http://www.abren. net/PrognoScan/) (19), and presented as a Kaplan-Meier plot, in which survival curves for high (red) and low (blue) expression groups dichotomized at the optimal cut-point were plotted. The P-values were adjusted for multiple correlation testing using the Miller and Siegmund formula (20), according to Prognoscan database and shown as a corrected P-value $\left(\mathrm{P}_{\text {cor }}\right)$. The threshold was adjusted to corrected $\mathrm{P}$-values at $<0.05$.
MethHC database analysis. The MethHC database was used in the analysis of SDPR DNA methylation alternation in cancer. MethHC (http://MethHC.mbc.nctu.edu.tw/) is a systematic database integrating DNA methylation data from The Cancer Genome Atlas (TCGA; https://cancergenome.nih.gov/abouttcga/policies/informedconsent), which includes $>6,000$ DNA methylation data generated by Illumina HumanMethylation450K BeadChip in 18 types of cancer. The methylation status of DNA was represented as $\beta$-values (0-1) (21), and the average $\beta$-value of $S D P R$ was presented as a boxplot by comparing the transcript expression in tumor samples and matched normal samples in all 18 types of cancer. To adjust the false discovery rate, the P-values were corrected using the B-H method in R software.

Methsurv database analysis. Methsurv database (https://biit. cs.ut.ee/methsurv) was utilized for survival analysis in different types of cancer based on $S D P R$ methylation patterns. In Methsurv, the gene methylation data was from TCGA Genome Data Analysis Center Firehose (http:/gdac.broadinstitute.org/) (22), using the HM450K array, which covers 486,428 CpGs. The methylation status of DNA was represented as $\beta$-values (0-1) (23). The methylation pattern was annotated by probes indicating subregions of the query gene, according to the annotation file (Human genome build 27) provided by Illumina [TSS to-200 nucleotides upstream of TSS ('TS200'); covering-200 to-1500 nucleotides upstream of TSS ('TSS1500'; first exon ('1st exon'); '5'UTR', 'body' and '3'UTR']. Clustering analysis was plotted and visualized using a heatmap by integrating Methsurv settings with ClustVis (https://biit.cs.ut. ee/clustvis/) (24). The survival analysis of each type of cancer between the low-methylated and the high-methylated groups in specific methylation sites was visualized using Kaplan-Meier plots. Multivariable survival analysis was performed using a Cox proportional-hazards model. Age and sex were used as covariates in the multivariable prediction models. The hazard ratio (HR) with $95 \% \mathrm{CI}$ was derived from Cox fitting. The goodness-of-fit of the Cox proportional hazard model was assessed using a likelihood-ratio (LR) test, and presented as an LR P-value. The methylation status of $S D P R$ in different LUAD clinical stage samples was shown as violin plots after grouping samples according to stage.

Identification of potential transcription factor for SDPR. The transcription start site (TSS) of SDPR was indicated by the University of California, Santa Cruz (UCSC) Genome Browser (http://genome.ucsc.edu), and the DNA sequence from 2,000 bp nucleotides upstream and 500 bp nucleotides downstream of the TSS was used as a potential promoter sequence for SDPR. PROMO at the ALGGEN server was subsequently used to identify the putative transcription factor binding sites in this promoter sequence (25). The co-expression profiles of the $S D P R$ gene in LUAD were identified and presented as the pattern of a heat map using the Oncomine database (17), in which the node correlation value is computed as the average of all pair-wise correlations among genes. The node correlation value $>0.5$ was used to define significant co-expressing genes. Finally, the intersection of the above two profiles was investigated in order to identify the potential transcription factor regulating $S D P R$ expression. 


\section{Results}

Downregulation of SDPR in various types of cancer. The expression of $S D P R$ is nearly ubiquitous in normal tissues and increased expression levels have been reported in the heart and lungs, while lower expression levels have been indicated in the kidney, the brain, the pancreas, skeletal muscle and the liver (8). To explore the gene expression alteration of SDPR in tumor tissues, the present study compared the mRNA levels of $S D P R$ in various unique tumor tissue datasets compared with normal tissue datasets from the Oncomine ${ }^{\mathrm{TM}}$ Platform (17). Consistent with previous studies, the analysis showed that the expression of SDPR was downregulated in breast $(1,11-13)$, kidney $(11,14)$ and prostate cancer $(11,15)$, and $\operatorname{SARC}(4,26)$. In addition, the present results identified that $S D P R$ is downregulated in bladder, lung, cervical, colorectal, gastric, ovarian and pancreatic cancer (Fig. 1).

Furthermore, gene expression levels of SDPR in cancer subtypes were estimated as a fold-change and gene rank (Table I). The fold change was from-44.083 (invasive ductal breast carcinoma) to-2.102 (cervical squamous cell carcinoma). The gene rank was between 6 and $1 \%$ (in the top \%). For instance, the entire subtype dataset of breast cancer showed $1 \%$ downregulated gene ranks. Downregulated gene expression of $S D P R$ was also observed in lung cancer (in top 3-1\%), pancreatic cancer (in top 1\%) and SARC (in top $4-1 \%$ ).

SDPR gene expression alteration and survival of patients. The PrognoScan platform, which integrates published cancer microarray datasets with clinical annotation (19), was used in the systematic meta-analysis and to determine the prognostic value of $S D P R$ in multiple datasets. Survival analysis consists of two steps, patient grouping and comparing the determined risks of these groups. Since gene expression is continuous data, the PrognoScan platform employed a minimum P-value approach for grouping patients in the survival analysis, which determines the optimal cut-off point in the continuous gene-expression measurement.

In the present study, PrognoScan indicated a significant association between microarray $S D P R$ expression and cancer prognosis in several tests: Brain (1/8), breast (10/40), lung (6/19) and soft tissue (1/1). In all these 18 tests, low SDPR expression was associated with poor survival (data not shown), suggesting its protective function in cancer malignancy. Decreased SDPR expression was significantly associated with decreased overall survival (OS) and relapse-free survival (RFS) of patients with breast cancer (Fig. 2A and B), consistent with a previous study (1). In addition, SDPR downregulation was significantly associated with OS and RFS in patients with lung cancer adenocarcinoma (Fig. 2C and D), OS in patients with glioma (Fig. 2E) and distant-recurrence free survival (DRFS) in liposarcoma (Fig. 2F).

SDPR is hypermethylated in specific types of cancer. In a previous next-generation sequencing study, $S D P R$ was not identified as a frequent mutational gene target (16) and it was also observed that $S D P R$ was epigenetically silenced in breast cancer cell lines (1). Therefore, the present study hypothesized that DNA methylation alteration, which is an important epigen-
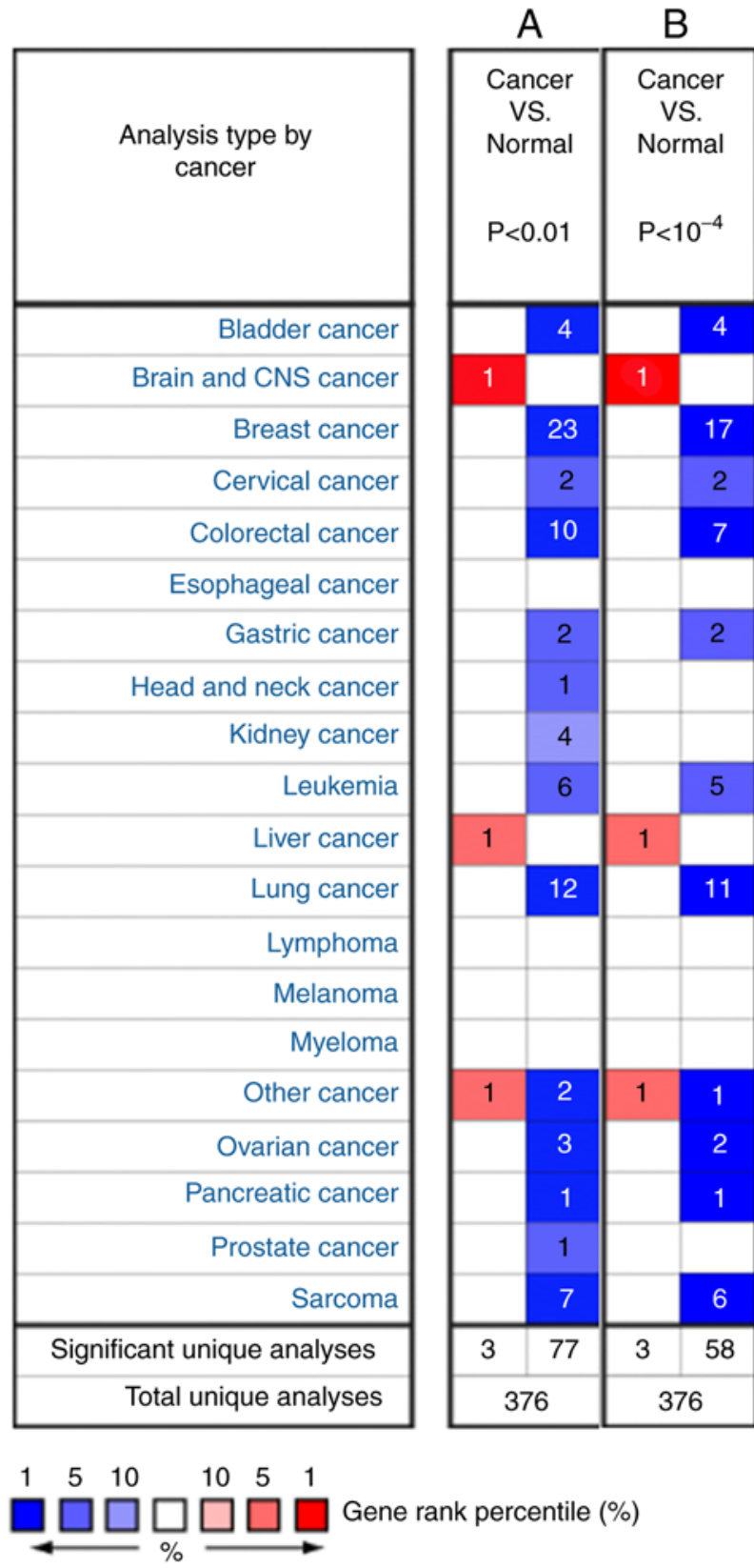

Figure 1. SDPR mRNA expression in different cancer types. (A) With a $\mathrm{P}$-value $<0.01$ threshold level, the comparison illustrated the number of datasets with SDPR mRNA overexpression (left column, red) and downregulation (right column, blue) in cancer compared with normal tissue. (B) With a P-value $<10^{-4}$ threshold level, the comparison of the number of datasets with $S D P R$ mRNA overexpression (left column, red) and downregulation (right column, blue) in cancer compared with normal tissue. The cell color was determined by the best gene rank percentile for the analyses within the cell. Common thresholds: Fold change of 2, gene rank at the top $10 \%$. SDPR, serum deprivation response; $\mathrm{CNS}$, central nervous system.

etic regulator for transcription, may be one of the mechanisms for $S D P R$ gene expression differences. In order to reveal the underlying molecular mechanisms responsible for $S D P R$ downregulation, MethHC was used to identify differential DNA methylation data between tumor and non-tumor tissues, which included 18 human types of cancer in $>6,000$ samples. MethHC is a database that systematically integrates DNA methylation and mRNA expression data from TCGA. The methylation profile of SDPR across 18 tumors is presented 

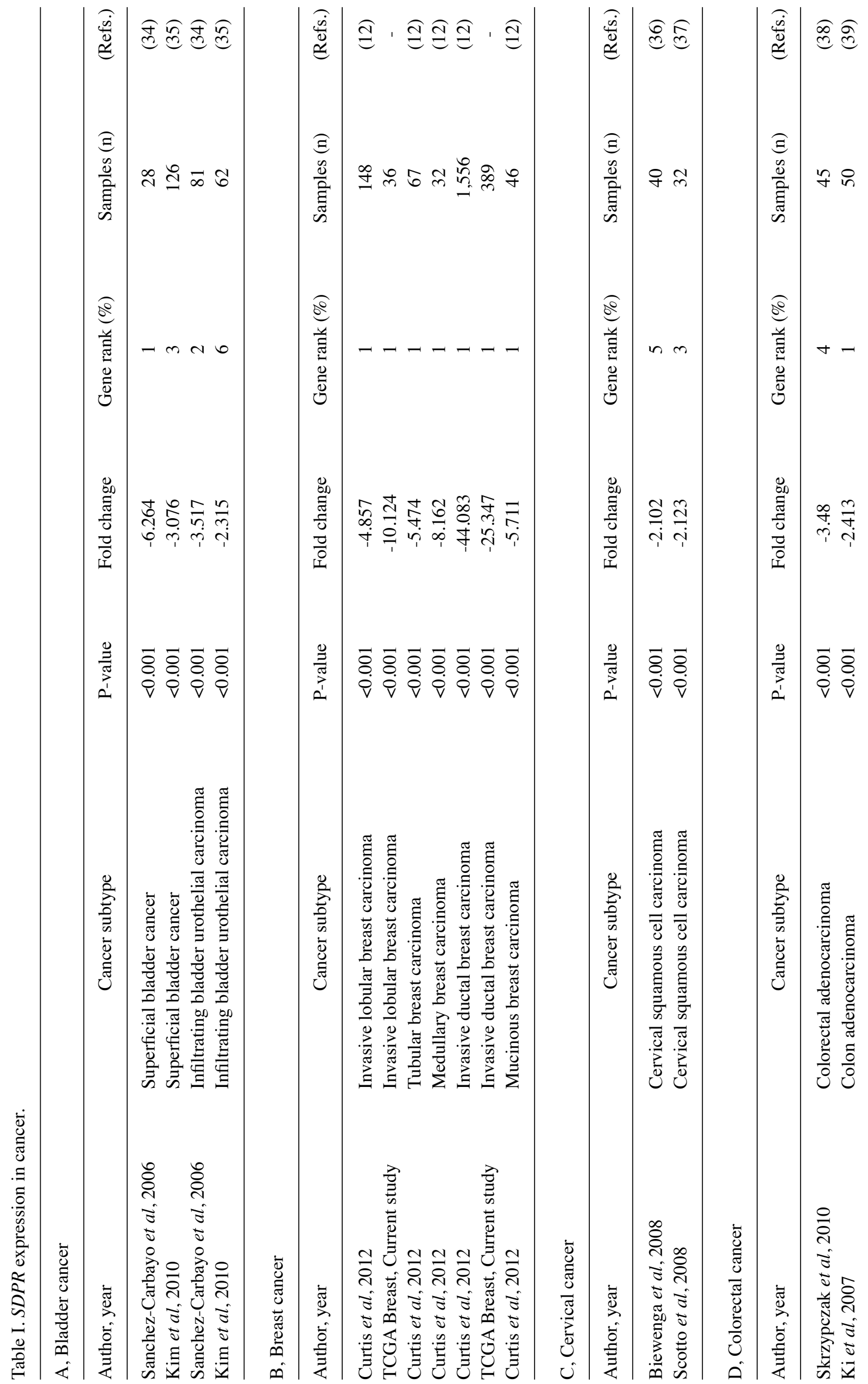

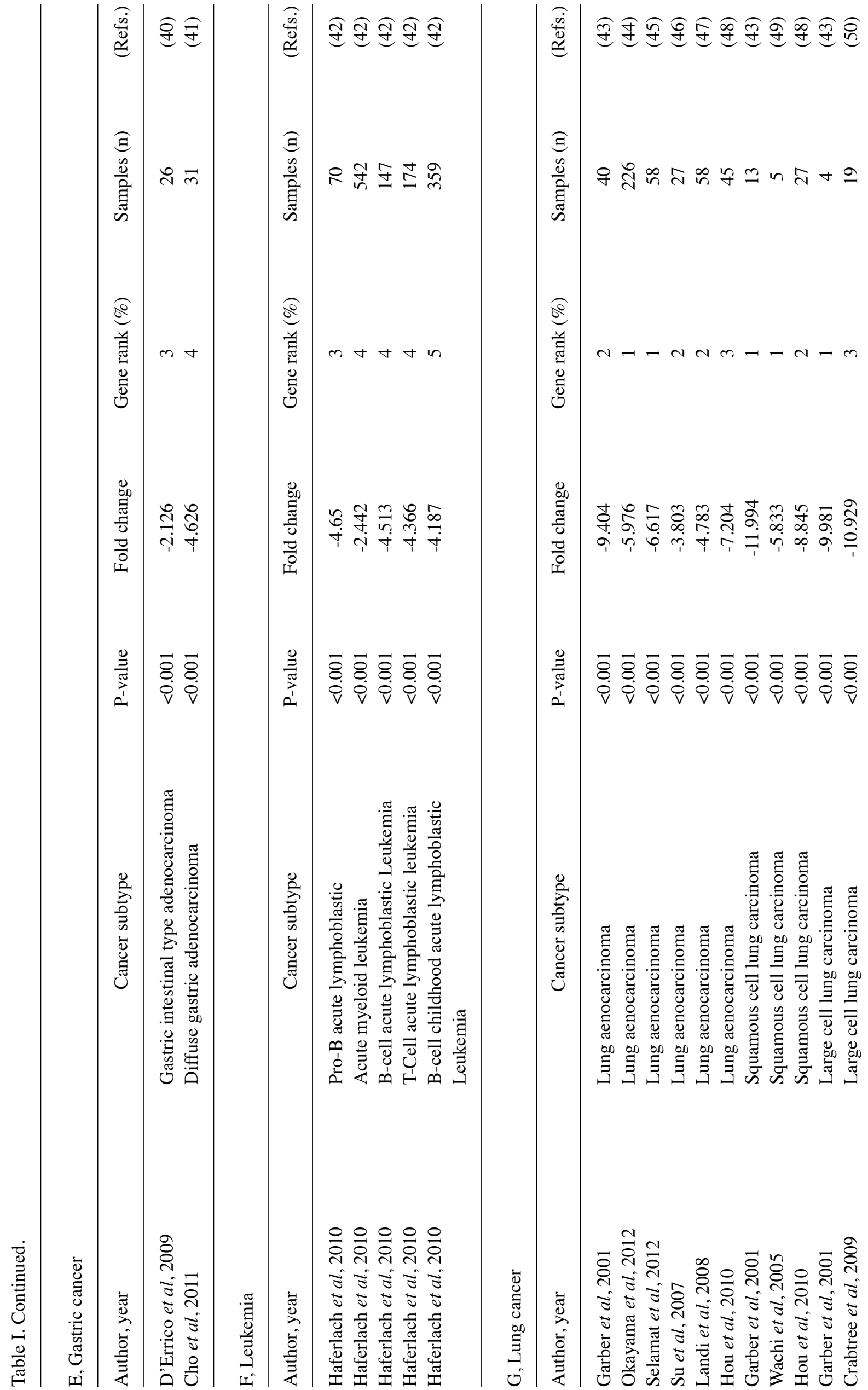

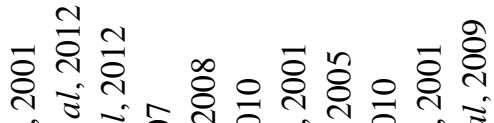

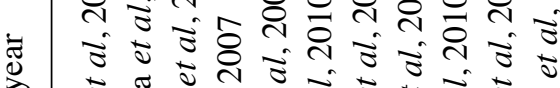

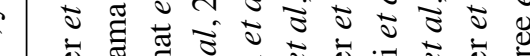

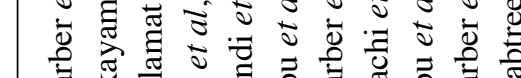

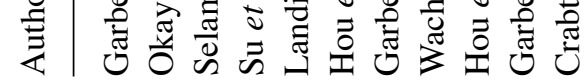




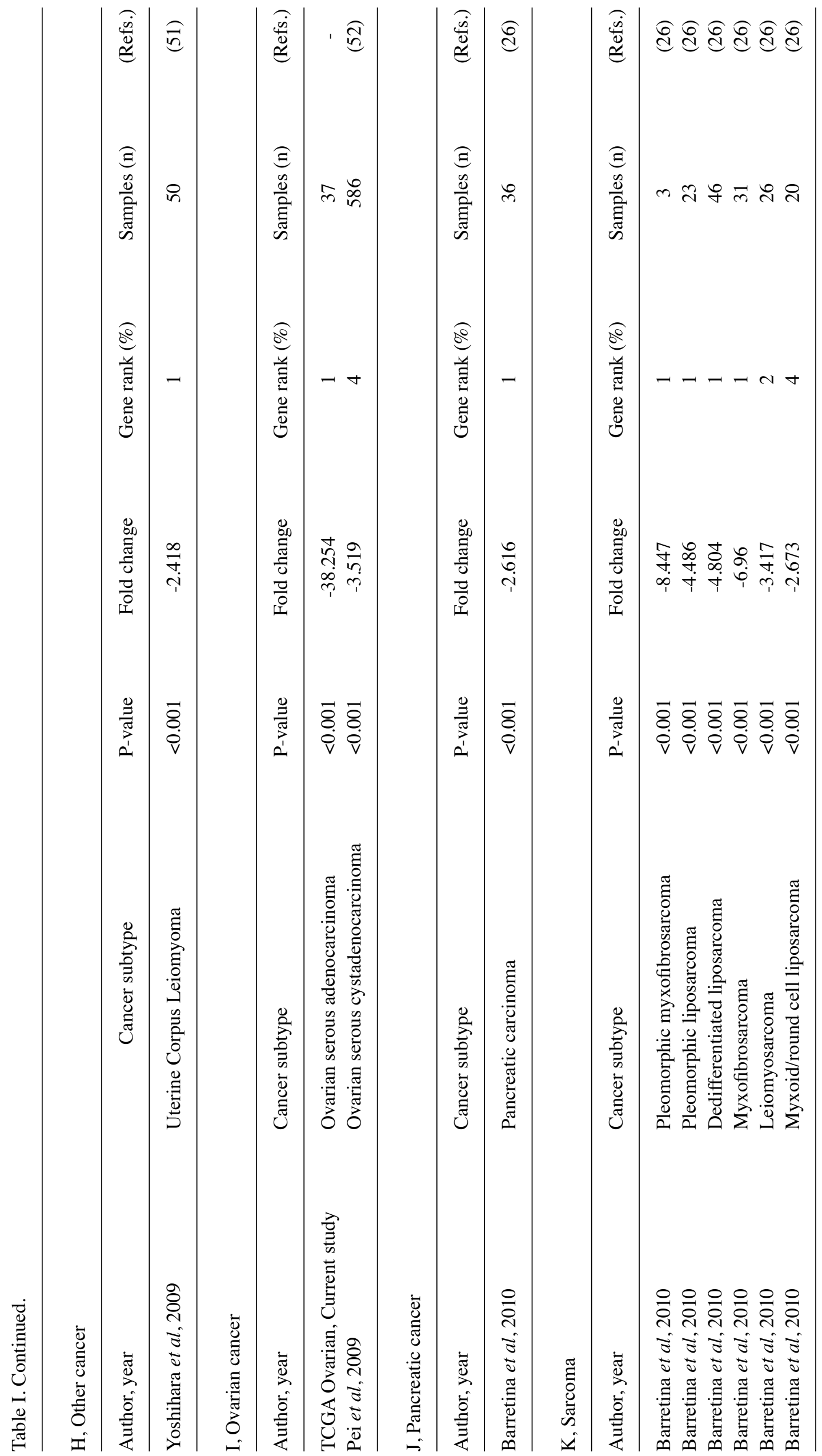


A

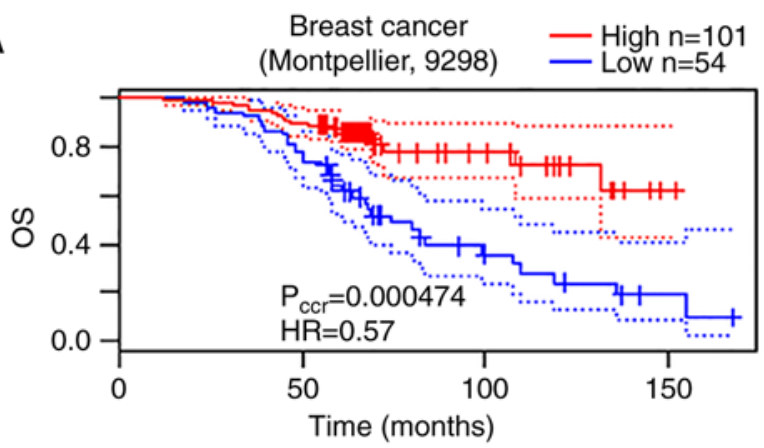

C

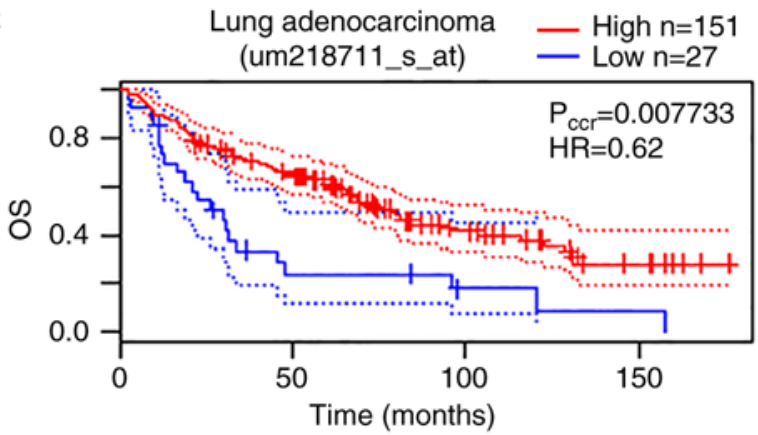

E

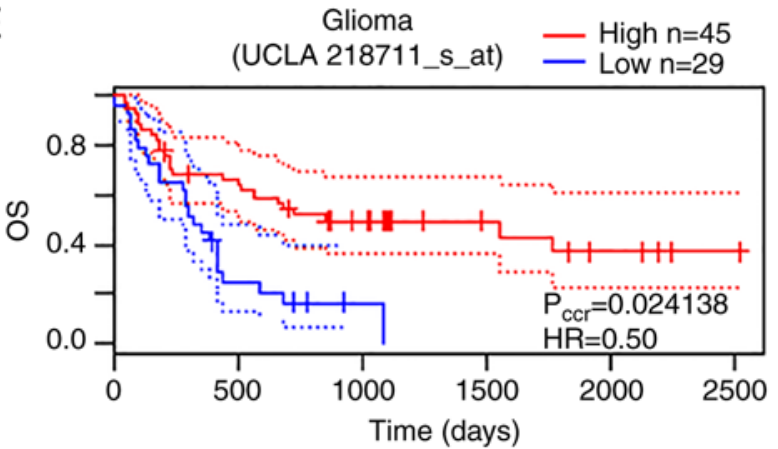

B

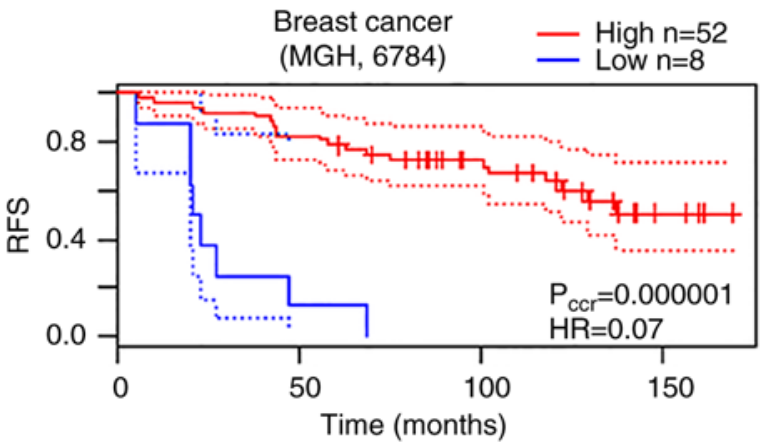

$\mathrm{D}$

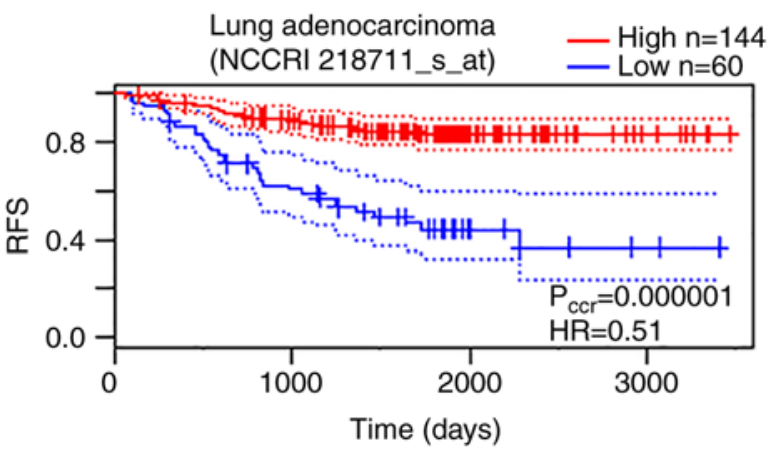

F

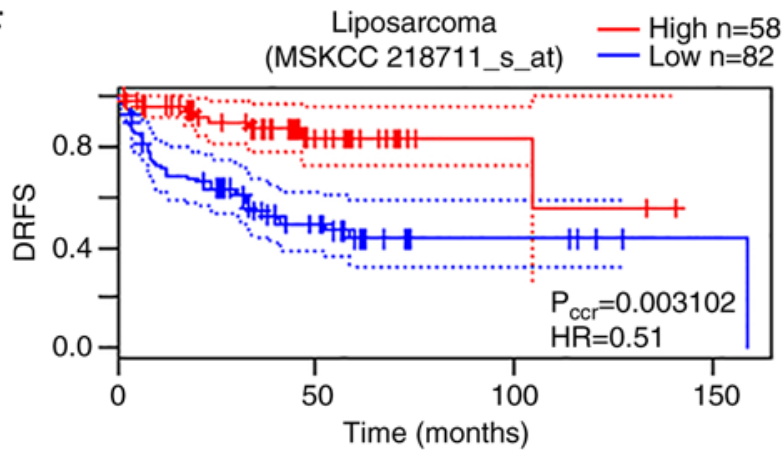

Figure 2. Survival curves comparing patients with high and low expressions of serum deprivation response were plotted from the PrognoScan database. (A) OS and (B) RFS survival curves comparing patients with high (red) and low (blue) expression in breast cancer were plotted from the PrognoScan database using the threshold of corrected P-value <0.05. (C) OS and (D) RFS survival curves comparing patients with high (red) and low (blue) expression in lung adenocarcinoma were plotted from the PrognoScan database using the threshold of corrected P-value $<0.05$. (E) OS survival curve comparing patients with high (red) and low (blue) expression in (E) glioma was plotted from the PrognoScan database as the threshold of corrected P-value <0.05. (F) DFRS survival curve comparing patients with high (red) and low (blue) expression in liposarcoma was plotted from the PrognoScan database as the threshold of corrected P-value $<0.05 .95 \%$ confidence intervals for each group are indicated by dotted lines. OS, overall survival; RFS, relapse-free survival; DRFS, distant-recurrence free survival; HR, hazard ratio; $\mathrm{P}_{\text {cor }}$, corrected $\mathrm{P}$-value.

in Fig. 3. In this profile, significantly SDPR gene methylation differences were observed in most types of cancer (17/18). Consistent with previously published experimental data, $S D P R$ was significantly hypermethylated in breast invasive carcinoma compared with normal breast tissues (1). In addition, SDPR was observed to be significantly hypermethylated in bladder urothelial carcinoma, cervical squamous cell carcinoma, head and neck squamous cell carcinoma, LUAD, lung squamous cell carcinoma (LUSC), pancreatic adenocarcinoma, prostate adenocarcinoma, SARC, skin cutaneous melanoma, stomach adenocarcinoma and uterine corpus endometrial carcinoma $(\mathrm{P}<0.005)$. Statistical differences were also found in colon adenocarcinoma, rectum adenocarcinoma and thyroid carcinoma $(\mathrm{P}<0.05)$. These results suggested that DNA methylation may be responsible for $S D P R$ downregulation in these types of cancer. As shown in Fig. 1, SDPR was downregulated in kidney cancer. However, significant $S D P R$ hypomethylation was found in both kidney renal clear cell carcinoma (KIRC) and kidney renal papillary cell carcinoma (KIRP) $(\mathrm{P}<0.005)$, suggesting the existence of other regulatory pathways.

Gene methylation of SDPR is associated with patient survival in $L U A D$ and $S A R C$. As downregulation of SDPR gene expression was observed in lung cancer and SARC, SDPR was indicated to be hypermethylated compared with normal tissues, and downregulation of SDPR expression was significantly associated with poor patient survival in LUAD and SARC, MethSurv was used to identify whether hypermethylation of $S D P R$ was associated with patient survival in LUAD or SARC. In addition, since the differential methylation levels at the $\mathrm{CpG}$ island are tissue-specific, the DNA methylation patterns were analyzed, taking into consideration the differential methylation levels in different gene subregions (Fig. 4A). MethSurv was the first database that indicated an association with overall 

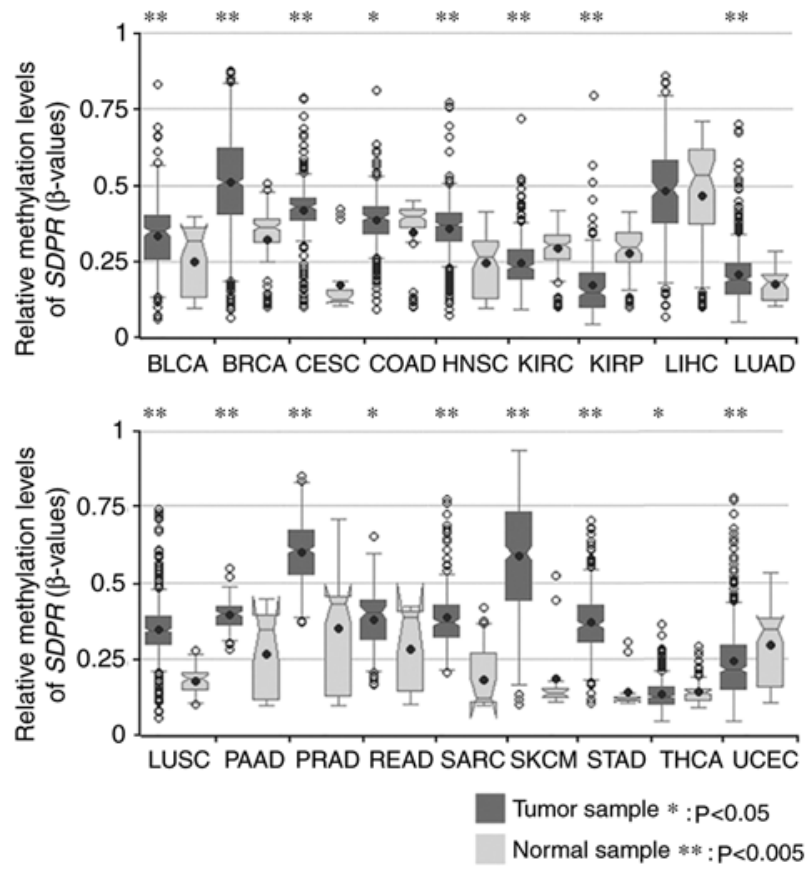

Figure 3. Methylation profiles of SDPR across tumors. The differential methylation statuses of each transcript of $S D P R$ in different tumor types are presented as boxplots. Tumor samples are in dark grey and are compared with normal samples in light grey. The shapes of notched boxplots indicated the characteristics of the data in each sub-dataset. P-values were adjusted using the Benjamini-Hochberg procedure. ${ }^{*} \mathrm{P}<0.05,{ }^{* *} \mathrm{P}<0.0005$ vs. normal sample. SDPR, serum deprivation response; BLCA, bladder urothelial carcinoma; BRCA, breast invasive carcinoma; CESC, cervical squamous cell carcinoma; COAD, colon adenocarcinoma; HNSC, head and neck squamous cell carcinoma; KIRC, kidney renal clear cell carcinoma; KIRP, kidney renal papillary cell carcinoma; LIHC, liver hepatocellular carcinoma; LUAD, lung adenocarcinoma; LUSC, lung squamous cell carcinoma; PAAD, pancreatic adenocarcinoma; PRAD, prostate adenocarcinoma; READ, rectum adenocarcinoma; SARC, sarcoma; SKCM, skin cutaneous adenocarcinoma; STAD, stomach adenocarcinoma; THCA, thyroid carcinoma; UCEC, uterine corpus endometrial carcinoma.

survival and DNA methylation patterns, in which the methylation levels are from TCGA methylation profile using the HM450k array. Based on the UCSC database, the CpG sites were grouped into gene subregions: 'TSS200', 'TSS1500', '1st exon', '5'UTR', 'body' and '3'UTR' (23). As shown in Fig. $4 \mathrm{~B}$ and $\mathrm{C}$, clustering visualization in the form of heat maps evaluated the association of methylation levels with the available patient characteristics and gene subregions in LUAD and SARC. According to the heatmaps, methylation sites cg18843739, cg10082589 and cg17809945 in the 'TSS1500' subregion showed differential methylation levels in patients with LUAD (461 samples). Similar to patients with SARC, the differently methylated sites were cg07488576 in the '1st Exon' subregion and cg18843739 in the 'TSS1500' subregion (261 samples).

MethSurv was used to identify which methylation sites were significantly associated with patient survival. By analyzing all 11 methylation sites in LUAD hypermethylation, it was indicated that two sites in LUAD (cg17809945 and cg10082589, located in the 'TSS1500' subregion of SDPR) were significantly associated with poor overall survival (Fig. 4D and E). In SARC, 2 out of 13 methylation sites (cg07488576 in the '1st Exon' subregion and $\operatorname{cg} 18843739$ in the 'TSS1500' subregion) were identified to be associated with poor survival (Fig. 4F and G). The results were consistent with the heatmap presented in Fig. $4 \mathrm{~B}$ and $\mathrm{C}$. In this analysis, CpG (cg10082589; HR=1.887; 95\% CI: 1.371-2.596; LR test $\mathrm{P}$-value $=0.001)$ was identified as the optimal survival methylation marker for LUAD (Fig. 4E) and CpG (cg07488576; HR=2.406; 95\% CI: $1.608-3.599$; LR test $P$-value $=0.00001$ ) was identified as the optimal survival methylation marker for SARC (Fig. 4F). In addition, when LUAD samples were grouped according to clinical stage, the methylation levels of cg17809945 (Fig. 4H) and cg10082589 (Fig. 4I) were indicated to be higher in late stages, suggesting that they may be involved in LUAD progression. However, no association between methylation levels of $\mathrm{CpG}$ sites and clinical stages was observed in SARC (data not shown).

Prediction of transcription factors regulating SDPR expression in $L U A D$. Since epigenetic patterns may not be the only reason for gene expression alternations in cancer, transcriptional regulation by deregulated transcription factors was investigated. The UCSC database was used to identify the promoter sequences of SDPR and PROMO was subsequently used at the ALGGEN server showing potential transcriptional factors on the promoter (Fig. 5A). Meanwhile, genes that were co-expressed with SDPR were analyzed using the Oncomine database, which were subsequently grouped into normal lung tissue and LUAD (Fig. 5B). By comparing the potential transcriptional factors with genes significantly co-expressed with SDPR (node correlation value $>0.5$ ), GATA binding protein 2 (GATA2; node correlation value $=0.807$ ) was identified as a potential transcription factor. GATA2 is a member of the GATA family, serves as a transcriptional activator during development and carcinogenesis, and was indicated to be epigenetically repressed in both human and mouse lung tumors (27). This result suggested that GATA2 may be a potential transcription factor regulating $S D P R$ gene expression in LUAD.

\section{Discussion}

$S D P R$ has been previously reported to show characteristic gene signatures in specific types of cancer, including breast $(1,2,11-13)$, thyroid $(3,28)$, oral $(5)$ and kidney $(11,14)$ cancer, and SARC $(4,26)$. In breast cancer, SDPR was identified as a novel tumor suppressor, which was significantly associated with patient survival $(1,2)$. However, a systemic profile of $S D P R$ alterations or analysis of its relevance in clinical outcomes in different types of cancer has yet to be performed, to the best of our knowledge. In the present study, $S D P R$ downregulation was observed in bladder, breast, lung, kidney, cervical, colorectal, gastric, ovarian and pancreatic cancer. Consistent with previous studies $(1,2), S D P R$ downregulation was significantly associated with patient survival in breast cancer. Furthermore, the analysis also indicated that decreased expression of $S D P R$ was significantly associated with poor OS and RFS in patients with adenocarcinoma of lung cancer, OS in patients with glioma and DRFS in liposarcoma.

In breast cancer, $S D P R$, which is partially silenced by DNA methylation, has been elucidated to execute an anti-metastatic function by promoting apoptosis (1), and depletion of 
A
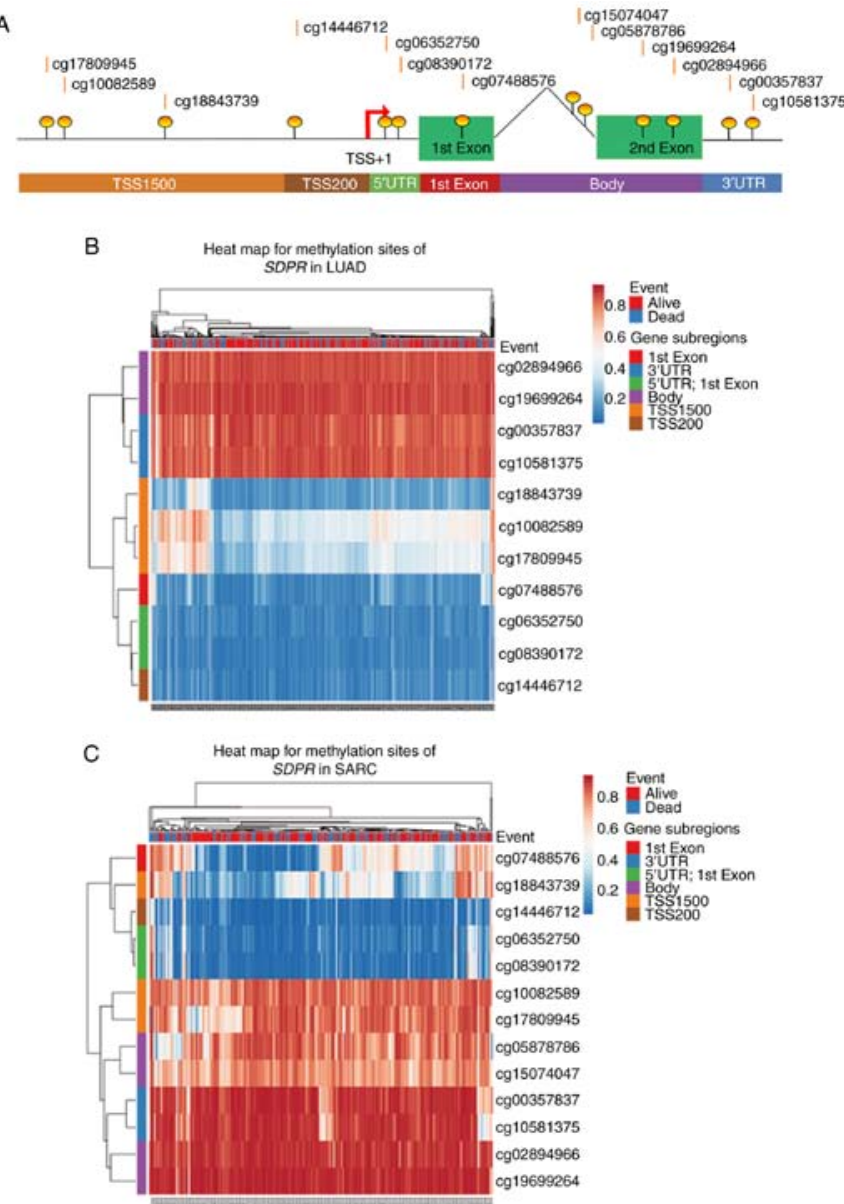
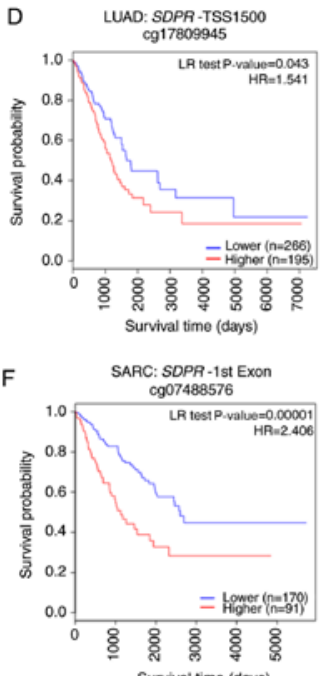

$\mathrm{H}$

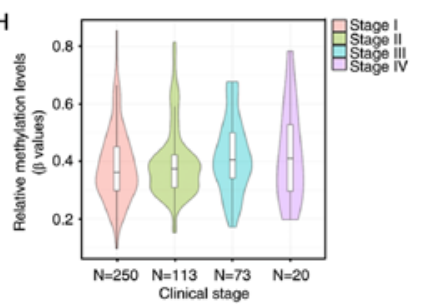

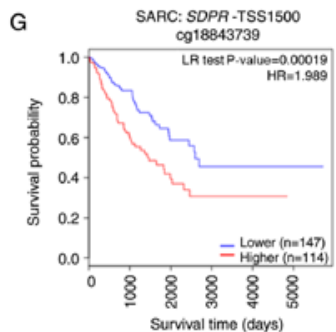
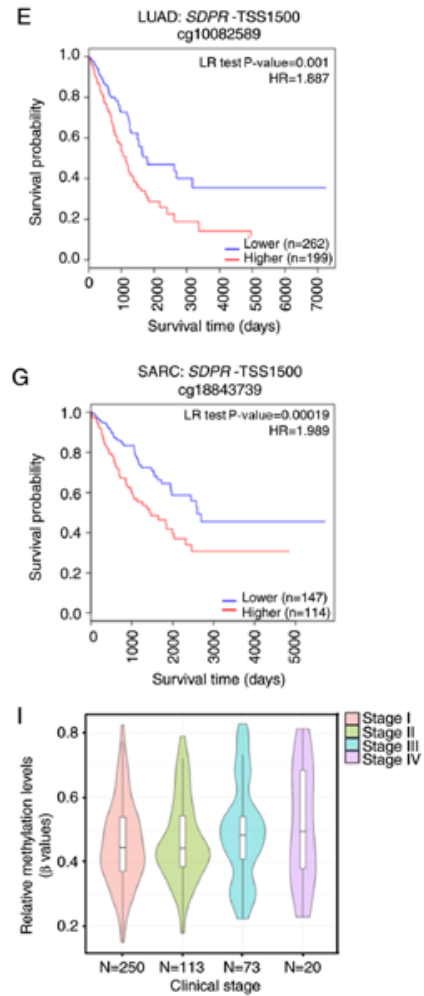

Figure 4. Gene methylation of SDPR is associated with patient survival in LUAD and SARC. (A) Diagram showing the relative location from the TSS for each CpG site of SDPR in Methsurv. Heat map depicting clustering analysis of the CpG methylation levels within the SDPR gene in (B) LUAD and (C) SARC. Methylation levels ( 0 represents fully unmethylated and 1 represents fully methylated) are shown as a continuous variable from blue to red color. Kaplan-Meier plot showed survival curve in higher (red) and lower (blue) methylation groups in methylation sites (D) cg17809945 and (E) cg10082859 in LUAD, and (F) cg07488576 and (G) cg18843739 in SARC. Violin plots showing the methylated levels of (H) cg17809945 and (I) cg10082859 among stage I, II, III and IV LUAD samples. The interquartile range and median methylation levels are shown in each violin plot as boxplots. SDPR, serum deprivation response; LUAD, lung adenocarcinoma; SARC, sarcoma; TSS, transcription start site; TSS1500, covering-200 to-1500 nucleotides upstream of TSS; TSS200, TSS to-200 nucleotides upstream of TSS; UTR, untranslated region; HR, hazard ratio; LR, log-likelihood ratio.

$S D P R$-induced EMT through TGF- $\beta$ signaling activation, according to previously published experimental studies $(1,2)$. To clarify why SDPR expression was altered in other types of cancer, the gene methylation level of $S D P R$ was subsequently profiled in 18 types of cancer and was compared with normal tissues. It was indicated to be significantly altered in 17 out of 18 types of cancer.

If DNA methylation of SDPR is involved in its downregulation in LUAD or SARC, it might be associated with patient survival. As not all of the methylation sites are responsible for gene expression, the most significant methylation sites require further investigation. Therefore, the present study analyzed the DNA methylation patterns in LUAD and SARC, considering the differential methylation levels in different gene subregions of SDPR. To the best of our knowledge, the present study for the first time, indicated that $\mathrm{CpG}(\operatorname{cg} 10082589)$ serves as the optimal survival methylation marker for LUAD, and $\mathrm{CpG}$ (cg07488576) serves as the optimal survival methylation marker for SARC. Furthermore, when LUAD samples were grouped according to clinical stage, the methylation level of CpG (cg10082589) and CpG (cg1780995) were higher in late stages, suggesting that they may be involved in LUAD progression.

In addition, by analyzing both the co-expressed genes with $S D P R$ and the putative transcription factor binding sites on SDPR, GATA2 was identified as a potential transcription factor for $S D P R$ transcription in LUAD. Transcriptional factor GATA2 regulates genes critical for embryonic development, self-renewal maintenance (29), functionality of blood-forming (30) and lymphatic vessel valve development (31). GATA2 has been reported to be frequently epigenetically repressed in both human and mouse lung tumors, and aberrant GATA2 methylation occurred early during lung carcinogenesis (27). GATA2 may serve a role in the downregulation of SDPR in LUAD.

Analysis of the associations between SDPR expression and DNA methylation with patient survival in LUSC was additionally performed. However, neither the OS nor disease-free survival of patients had been observed to be significantly associated with SDPR expression alternations, according to the Prognoscan database. Furthermore, similar to LUAD, the same $13 \mathrm{CpG}$ sites grouped in gene subregions based on the 
A

\begin{tabular}{|c|}
\hline \multirow{2}{*}{$\frac{H}{4} \frac{H N F-3 \alpha[T 02512]}{\text { YY1 [T00915] }}$} \\
\hline \\
\hline 8 FOXP3 [T04280] \\
\hline 12 HOXD10 [T01425] \\
\hline 16 MEF-2A [T01005] \\
\hline 20 PR B [T00696] \\
\hline 24 STAT4 [T01577] \\
\hline 28 GATA-2 [T00308] \\
\hline 32 IRF-2 [T01491] \\
\hline 36 ENKTF-1 [T00255 \\
\hline 40 NF-1 [T00539] \\
\hline 44 ER- $\alpha$ [T00261] \\
\hline 48 CTF [T00174] \\
\hline 52 PU.1 [T02068] \\
\hline 56 ETF [T00270] \\
\hline 60 NF-AT1 [T01948] \\
\hline 64 AR [T00040] \\
\hline 68 Egr-3 [T00243] \\
\hline 72 EBF [T05427] \\
\hline 76 RBP-Jк [T01616] \\
\hline
\end{tabular}

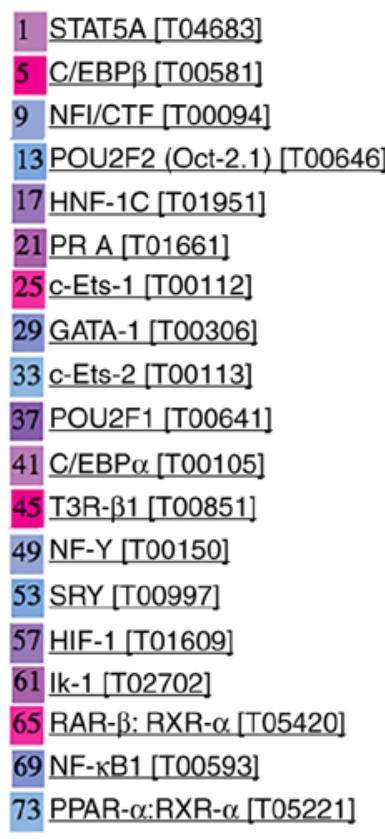

\begin{tabular}{|c|}
\hline 3 TFII-I [T00824] \\
\hline 7 р53 [т00671] \\
\hline 11 HOXD9 [T01424] \\
\hline 15 GR [T05076] \\
\hline 19 HNF-1B [T01950] \\
\hline 23 NF-AT2 [T01945] \\
\hline 27 RXR- $\alpha$ [T01345] \\
\hline 31 GR- $\alpha$ [T00337] \\
\hline 35 NF-AT1 [T00550] \\
\hline 39 AP-2 $\alpha$ A [T00035] \\
\hline 43 PEA3 [T00685] \\
\hline 47 LEF-1 [T02905] \\
\hline 51 ELF-1 [T01113] \\
\hline 55 SRF [T00764] \\
\hline 59 USF1 [T00874] \\
\hline 63 E2F-1 [T01542] \\
\hline 67 PXR-1:RXR- $\alpha$ [T05671] \\
\hline 71 MAZ [T00490] \\
\hline 75 USF2 [T00878] \\
\hline
\end{tabular}

\footnotetext{
$5^{\prime}$
}

TFs binding sites on SDPR promoter

B

Genes coexpressed with SDPR

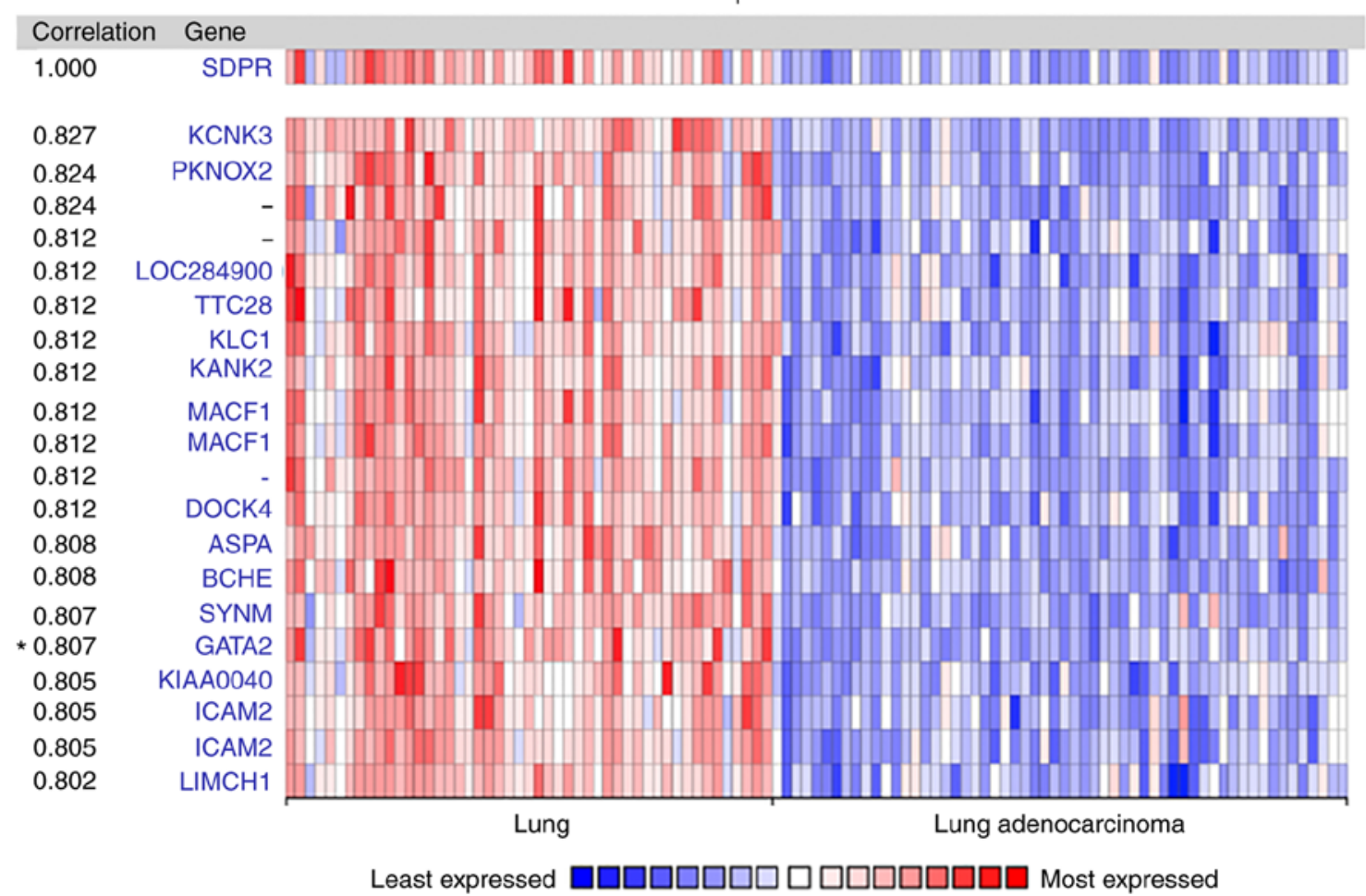

Figure 5. Prediction of TFs regulating SDPR expression. (A) TFs predicted using PROMO in the SDPR promoter sequence. The numbers in the square brackets represent the annotation for each TF in the PROMO database. (B) SDPR is coexpressed with the indicated genes across a panel of 58 lung adenocarcinoma and 49 normal samples. Normalized gene expressing levels are shown from least expressed (blue) to most expressed (red) within each row. The asterisks indicate the GATA2 results. SDPR, serum deprivation response; TF, transcription factor; GATA2, GATA binding protein 2.

UCSC database were analyzed using MethSurv. However, the results indicated no significant association between DNA methylation data and patient survival (data not shown). Since gene expression patterns differ in the subtypes of lung cancer, this may provide novel insight for the examination of potential mechanisms of $S D P R$ function in LUAD.
$S D P R$ was also downregulated in kidney cancer. Significant hypomethylation was found in both KIRC and KIRP, suggesting other regulatory pathways. By comparing methylation patterns with patient survival in KIRC and KIRP, the present study indicated that hypomethylation in the 'TSS200' and 'TSS1500' subregions of SDPR was 
significantly associated with longer survival time, while hypomethylation in the gene 'body' and the ' 3 'UTR' subregions was associated with poor OS (data not shown). The function of DNA methylation status seems to vary in context; this may be due to hypermethylation in the promoter region inducing the downregulation of gene expression, while hypermethylation in the gene body may not block and may even stimulate transcription elongation, and the gene body methylation may have an impact on splicing (32). In addition, long non-coding RNA (lncRNA) SDPR-antisense (SDPR-AS) has been verified to be co-expressed with SDPR, and elevated IncRNA SDPR-AS increases the OS in renal cell carcinoma, suggesting the possibility that lncRNAs may serve a regulatory role in the $S D P R$ pathway (33).

The specific methylation CpG sites, which are significantly associated with patient survival, require further study in order to verify the present results, such as pyrosequencing and reverse transcription-quantitative PCR. The potential transcription factor GATA2 should also be experimentally investigated to determine whether it is responsible in SDPR transactivation. Despite taking age and sex into consideration as covariates in survival analysis based on the Methsurv database, due to the lack of available clinical data, the survival analysis based on PrognoScan is univariable. Since several factors, such as co-morbidities, performance status and treatments, may potentially affect the prognosis of patients with cancer, it is important to consider all potential relevant specific features for specific cancer types in future clinical investigations of $S D P R$.

In summary, the present study suggested that the role of $S D P R$ as a tumor suppressor may have broader clinical relevance beyond breast cancer. The present study on $S D P R$ may help to examine its underlying molecular mechanism in cancer progression, reveal novel perspectives for prognostic evaluation in specific cancer, and provide insight for further research in the field.

\section{Acknowledgements}

Not applicable.

\section{Funding}

The present study was supported by Foundation of Sichuan Educational Committee, People's Republic of China (grant nos. 17ZA0164 and 18CZ0010) and Foundation of Chengdu University of Traditional Chinese Medicine, China (grant nos. ZRQN1660 and CGZH1709).

\section{Availability of data and materials}

The datasets used and/or analyzed during the current study are available from the corresponding author on reasonable request.

\section{Authors' contributions}

YW and YL designed the present study. YW analyzed the gene expressing data, patient survival data and the potential transcriptional factors of SDPR. ZS analyzed the co-expressing genes with SDPR. YL and PL analyzed the gene methylation data. YW wrote the manuscript and YL revised it. All authors read and approved the final manuscript.

\section{Ethics approval and consent to participate}

Not applicable.

\section{Patient consent for publication}

Not applicable.

\section{Competing interests}

The authors declare that they have no competing interests.

\section{References}

1. Ozturk S, Papageorgis $\mathrm{P}$, Wong CK, Lambert AW Abdolmaleky HM, Thiagalingam A, Cohen HT and Thiagalingam S: SDPR functions as a metastasis suppressor in breast cancer by promoting apoptosis. Proc Natl Acad Sci USA 113: 638-643, 2016.

2. Tian Y, Yu Y, Hou LK, Chi JR, Mao JF, Xia L, Wang X, Wang P and $\mathrm{Cao} \mathrm{XC}$ : Serum deprivation response inhibits breast cancer progression by blocking transforming growth factor- $\beta$ signaling. Cancer Sci 107: 274-280, 2016.

3. Poma AM, Giannini R, Piaggi P, Ugolini C, Materazzi G, Miccoli P, Vitti P and Basolo F: A six-gene panel to label follicular adenoma, low- and high-risk follicular thyroid carcinoma. Endocr Connect 7: 124-132, 2018.

4. Codenotti S, Vezzoli M, Poliani PL, Cominelli M, Monti E and Fanzani A: Cavin-2 is a specific marker for detection of well-differentiated liposarcoma. Biochem Biophys Res Commun 493: 660-665, 2017.

5. Unozawa M, Kasamatsu A, Higo M, Fukumoto C, Koyama T, Sakazume T, Nakashima D, Ogawara K, Yokoe H, Shiiba M, et al: Cavin-2 in oral cancer: A potential predictor for tumor progression. Mol Carcinog 55: 1037-1047, 2016.

6. Hansen CG, Bright NA, Howard G and Nichols BJ: SDPR induces membrane curvature and functions in the formation of caveolae. Nat Cell Biol 11: 807-814, 2009.

7. Nassar ZD and Parat MO: Cavin family: New players in the biology of caveolae. Int Rev Cell Mol Biol 320: 235-305, 2015.

8. Gustincich S, Vatta P, Goruppi S, Wolf M, Saccone S, Della Valle G, Baggiolini $M$ and Schneider C: The human serum deprivation response gene (SDPR) maps to $2 \mathrm{q} 32-\mathrm{q} 33$ and codes for a phosphatidylserine-binding protein. Genomics 57: 120-129, 1999.

9. Baig A, Bao X, Wolf $M$ and Haslam RJ: The platelet protein kinase $\mathrm{C}$ substrate pleckstrin binds directly to SDPR protein. Platelets 20: 446-457, 2009.

10. Gupta R, Toufaily C and Annabi B: Caveolin and cavin family members: Dual roles in cancer. Biochimie 107: 188-202, 2014.

11. Li X, Jia Z, Shen Y, Ichikawa H, Jarvik J, Nagele RG and Goldberg GS: Coordinate suppression of Sdpr and Fhl1 expression in tumors of the breast, kidney, and prostate. Cancer Sci 99: 1326-1333, 2008.

12. Curtis C, Shah SP, Chin SF, Turashvili G, Rueda OM, Dunning MJ, Speed D, Lynch AG, Samarajiwa S, Yuan Y, et al: The genomic and transcriptomic architecture of 2,000 breast tumours reveals novel subgroups. Nature 486: 346-352, 2012.

13. Bai L, Deng X, Li Q, Wang M, An W, Deli A, Gao Z, Xie Y, Dai Y and Cong YS: Down-regulation of the cavin family proteins in breast cancer. J Cell Biochem 113: 322-328, 2012.

14. Gianazza E, Chinello C, Mainini V, Cazzaniga M, Squeo V, Albo G, Signorini S, Di Pierro SS, Ferrero S, Nicolardi S, et al: Alterations of the serum peptidome in renal cell carcinoma discriminating benign and malignant kidney tumors. J Proteomics 76: 125-140, 2012.

15. Altintas DM, Allioli N, Decaussin M, de Bernard S, Ruffion A, Samarut J and Vlaeminck-Guillem V: Differentially expressed androgen-regulated genes in androgen-sensitive tissues reveal potential biomarkers of early prostate cancer. PLoS One 8: e66278, 2013 
16. Vogelstein B, Papadopoulos N, Velculescu VE, Zhou S, Diaz LA Jr and Kinzler KW: Cancer genome landscapes. Science 339: 1546-1558, 2013.

17. Rhodes DR, Kalyana-Sundaram S, Mahavisno V, Varambally R, Yu J, Briggs BB, Barrette TR, Anstet MJ, Kincead-Beal C, Kulkarni P, et al: Oncomine 3.0: Genes, pathways, and networks in a collection of 18,000 cancer gene expression profiles Neoplasia 9: 166-180, 2007.

18. Benjamini Y and Hochberg Y: Controlling the false discovery rate: A practical and powerful approach to multiple testing. J R Statist Soc B 57: 289-300, 1995.

19. Mizuno H, Kitada K, Nakai K and Sarai A: PrognoScan: A new database for meta-analysis of the prognostic value of genes. BMC Med Genomics 2: 18, 2009.

20. Miller R and Siegmund D: Maximally selected chi square statistics. Biometrics 38: 1011-1016, 1982.

21. Huang WY, Hsu SD, Huang HY, Sun YM, Chou CH, Weng SL and Huang HD: MethHC: A database of DNA methylation and gene expression in human cancer. Nucleic Acids Res 43 (Database Issue): D856-D861, 2015.

22. Broad Institute TCGA Genome Data Analysis Center (2016): Firehose stddata_2016_01_28 run. Broad Institute of MIT and Harvard. Doi: $10 . \overline{7908 / C 11 G 0 K M 9 . ~}$

23. Modhukur V, Iljasenko T, Metsalu T, Lokk K, Laisk-Podar T and Vilo J: MethSurv: A web tool to perform multivariable survival analysis using DNA methylation data. Epigenomics 10: 277-288, 2018.

24. Metsalu T and Vilo J: ClustVis: A web tool for visualizing clustering of multivariate data using principal component analysis and heatmap. Nucleic Acids Res 43W: W566-W570, 2015.

25. Farré D, Roset R, Huerta M, Adsuara JE, Roselló L, Albà MM and Messeguer X: Identification of patterns in biological sequences at the ALGGEN server: PROMO and MALGEN. Nucleic Acids Res 31: 3651-3653, 2003.

26. Barretina J, Taylor BS, Banerji S, Ramos AH, Lagos-Quintana M, Decarolis PL, Shah K, Socci ND, Weir BA, Ho A, et al: Subtype-specific genomic alterations define new targets for soft-tissue sarcoma therapy. Nat Genet 42: 715-721, 2010.

27. Tessema M, Yingling CM, Snider AM, Do K, Juri DE, Picchi MA, Zhang X, Liu Y, Leng S, Tellez CS and Belinsky SA: GATA2 is epigenetically repressed in human and mouse lung tumors and is not requisite for survival of KRAS mutant lung cancer. J Thorac Oncol 9: 784-793, 2014

28. Borup R, Rossing M, Henao R, Yamamoto Y, Krogdahl A Godballe C, Winther O, Kiss K, Christensen L, Høgdall E, et al: Molecular signatures of thyroid follicular neoplasia. Endocr Relat Cancer 17: 691-708, 2010.

29. Krendl C, Shaposhnikov D, Rishko V, Ori C, Ziegenhain C, Sass S, Simon L, Müller NS, Straub T, Brooks KE, et al: GATA2/3-TFAP2A/C transcription factor network couples human pluripotent stem cell differentiation to trophectoderm with repression of pluripotency. Proc Natl Acad Sci USA 114 E9579-E9588, 2017.

30. de Pater E, Kaimakis P, Vink CS, Yokomizo T, Yamada-Inagawa T, van der Linden R, Kartalaei PS, Camper SA, Speck N and Dzierzak E: Gata2 is required for HSC generation and survival. J Exp Med 210: 2843-2850, 2013.

31. Kazenwadel J, Betterman KL, Chong CE, Stokes PH, Lee YK, Secker GA, Agalarov Y, Demir CS, Lawrence DM, Sutton DL, et al: GATA2 is required for lymphatic vessel valve development and maintenance. J Clin Invest 125: 2979-2994, 2015.

32. Jones PA: Functions of DNA methylation: Islands, start sites, gene bodies and beyond. Nat Rev Genet 13: 484-492, 2012.

33. Ni W, Song E, Gong M, Li Y, Yao J and An R: Downregulation of lncRNA SDPR-AS is associated with poor prognosis in renal cell carcinoma. Onco Targets Ther 10: 3039-3047, 2017.

34. Sanchez-Carbayo M, Socci ND, Lozano J, Saint F and Cordon-Cardo C: Defining molecular profiles of poor outcome in patients with invasive bladder cancer using oligonucleotide microarrays. J Clin Oncol 24: 778-789, 2006.

35. Kim WJ, Kim EJ, Kim SK, Kim YJ, Ha YS, Jeong P, Kim MJ, Yun SJ, Lee KM, Moon SK, et al: Predictive value of progression-related gene classifier in primary non-muscle invasive bladder cancer. Mol Cancer 9: 3, 2010.

36. Biewenga P, Buist MR, Moerland PD, Ver Loren van Themaat E, van Kampen AH, ten Kate FJ and Baas F: Gene expression in early stage cervical cancer. Gynecol Oncol 108: 520-526, 2008.
37. Scotto L, Narayan G, Nandula SV, Arias-Pulido H, Subramaniyam S, Schneider A, Kaufmann AM, Wright JD, Pothuri B, Mansukhani M and Murty VV: Identification of copy number gain and overexpressed genes on chromosome arm 20c by an integrative genomic approach in cervical cancer: Potential role in progression. Genes Chromosomes Cancer 47: 755-765, 2008.

38. Skrzypczak M, Goryca K, Rubel T, Paziewska A, Mikula M, Jarosz D, Pachlewski J, Oledzki J and Ostrowski J: Modeling oncogenic signaling in colon tumors by multidirectional analyses of microarray data directed for maximization of analytical reliability. PLoS One 5: pii: e13091, 2010.

39. Ki DH, Jeung HC, Park CH, Kang SH, Lee GY, Lee WS, Kim NK, Chung HC and Rha SY: Whole genome analysis for liver metastasis gene signatures in colorectal cancer. Int $\mathrm{J}$ Cancer 121: 2005-2012, 2007.

40. D'Errico M, de Rinaldis E, Blasi MF, Viti V, Falchetti M, Calcagnile A, Sera F, Saieva C, Ottini L, Palli D, et al: Genome-wide expression profile of sporadic gastric cancers with microsatellite instability. Eur J Cancer 45: 461-469, 2009.

41. Cho JY, Lim JY, Cheong JH, Park YY, Yoon SL, Kim SM, Kim SB, Kim H, Hong SW, Park YN, et al: Gene expression signature-based prognostic risk score in gastric cancer. Clin Cancer Res 17: 1850-1857, 2011.

42. Haferlach T, Kohlmann A, Wieczorek L, Basso G, Kronnie GT, Béné MC, De Vos J, Hernández JM, Hofmann WK, Mills KI, et al: Clinical utility of microarray-based gene expression profiling in the diagnosis and subclassification of leukemia: Report from the international microarray innovations in leukemia study group. J Clin Oncol 28: 2529-2537, 2010.

43. Garber ME, Troyanskaya OG, Schluens K, Petersen S, Thaesler Z, Pacyna-Gengelbach M, van de Rijn M, Rosen GD, Perou CM, Whyte RI, et al: Diversity of gene expression in adenocarcinoma of the lung. Proc Natl Acad Sci USA 98: 13784-13789, 2001.

44. Okayama H, Kohno T, Ishii Y, Shimada Y, Shiraishi K, Iwakawa R, Furuta K, Tsuta K, Shibata T, Yamamoto S, et al: Identification of genes upregulated in ALK-positive and EGFR/KRAS/ALK-negative lung adenocarcinomas. Cancer Res 72: 100-111, 2012.

45. Selamat SA, Chung BS, Girard L, Zhang W, Zhang Y, Campan M, Siegmund KD, Koss MN, Hagen JA, Lam WL, et al: Genome-scale analysis of DNA methylation in lung adenocarcinoma and integration with mRNA expression. Genome Res 22: 1197-1211, 2012.

46. Su LJ, Chang CW, Wu YC, Chen KC, Lin CJ, Liang SC, Lin CH, Whang-Peng J, Hsu SL, Chen CH and Huang CY: Selection of DDX5 as a novel internal control for Q-RT-PCR from microarray data using a block bootstrap re-sampling scheme. BMC Genomics 8: 140, 2007.

47. Landi MT, Dracheva T, Rotunno M, Figueroa JD, Liu H, Dasgupta A, Mann FE, Fukuoka J, Hames M, Bergen AW, et al: Gene expression signature of cigarette smoking and its role in lung adenocarcinoma development and survival. PLoS One 3: e1651, 2008.

48. Hou J, Aerts J, den Hamer B, van Ijcken W, den Bakker M, Riegman P, van der Leest C, van der Spek P, Foekens JA, Hoogsteden HC, et al: Gene expression-based classification of non-small cell lung carcinomas and survival prediction. PLoS One 5: e10312, 2010.

49. Wachi S, Yoneda K and Wu R: Interactome-transcriptome analysis reveals the high centrality of genes differentially expressed in lung cancer tissues. Bioinformatics 21: 4205-4208, 2005.

50. Crabtree JS, Jelinsky SA, Harris HA, Choe SE, Cotreau MM, Kimberland ML, Wilson E, Saraf KA, Liu W, McCampbell AS, et al: Comparison of human and rat uterine leiomyomata: Identification of a dysregulated mammalian target of rapamycin pathway. Cancer Res 69: 6171-6178, 2009.

51. Yoshihara K, Tajima A, Komata D, Yamamoto T, Kodama S, Fujiwara H, Suzuki M, Onishi Y, Hatae M, Sueyoshi K, et al: Gene expression profiling of advanced-stage serous ovarian cancers distinguishes novel subclasses and implicates ZEB2 in tumor progression and prognosis. Cancer Sci 100: 1421-1428, 2009.

52. Pei H, Li L, Fridley BL, Jenkins GD, Kalari KR, Lingle W, Petersen G, Lou Z and Wang L: FKBP51 affects cancer cell response to chemotherapy by negatively regulating Akt. Cancer Cell 16: 259-266, 2009. 